\title{
Predictive Holographic Neural Network Control of 2 DOF Magnetic Levitation Device
}

\author{
S. Darenfed, \\ Universite de Moncton \\ darenfs@umoncton.ca
}

\author{
G. Sridharan \\ XdTech Ltd. \\ gs@xdtech.com
}

\begin{abstract}
The objective herein is to demonstrate the feasibility of a real-time digital control of a 2 DOF magnetic levitation device for modeling and controls education, with emphasis on predictive holographic neural network control. The plant of interest is a magnetic levitation device that is nonlinear and open-loop unstable. In this application, the reference model of the plant is a neural network that has an embedded nominal linear model in the network weights. The control based on the linear model provides initial stability at the beginning of network training. In using a holographic neural network the control laws are nonlinear and online adaptation of the model is possible to capture unmodeled or time-varying dynamics. Such an environment provides for experimentation, data collection, system identification and novel control strategy implementation. The environment is used to implement predictive holographic neural networks with real-time dynamic weight tuning and controller performance comparison under various trajectories input. The educational features of this environment are being tested in a senior control engineering classroom setting.
\end{abstract}

\section{Introduction}

To make the implementation of advanced control algorithms feasible in an educational setting, a hardware-in-the-loop (HIL) environment is considered. The coupling of a HIL device with a modeling and simulation environment is a mean to reinforce classroom concepts, observe system performance following changes in control laws and system dynamics in real time. Matlab/Simulink [1], a fifth generation software environment in wide use in engineering courses, provides students with a familiar interface to experiment and gain insight into advanced concepts. The Real Time Workshop (RTW) toolbox, coupled with a low cost data acquisition card (DAC) on a personal computer allow access to real time analysis and design without the burden of low level software development. In the next section the experimental eetup will be described followed by discussions of the holographic neural predictive control, the MAGLEV2 model, results and conclusions

\section{Experimental Setup}

Magnetic levitation and magnetic bearings are growing in popularity as well as in engineering usage in diverse fields, from large turbo machinery to spacebased structures. These devices are used in both rotary applications and linear motion applications. Magnetic suspension systems are especially useful in applications where high vacuum ambient is present because use of conventional lubricants is to be avoided. The reliability and microminiaturization of electronic devices have resulted in magnetic bearings for varied industrial applications. What makes a magnetic levitation an interesting and challenging device is its basic unstable nature. That is, these devices are open-loop unstable, in control theory parlance. Hence a feedback control mechanism is required to enable the systems operate in stable fashion. The device here has an even more intricate formulation. It operates in a variant mode: Permanent magnet augmentation in both stator assembly and suspended member. While this variant leads to lower operating current, more complex modeling results. The MAGLEV2 device used for this work has two coils that are positioned side by side to suspend two cylindrical objects connected by a non ferrous rod as shown in Figure 1.

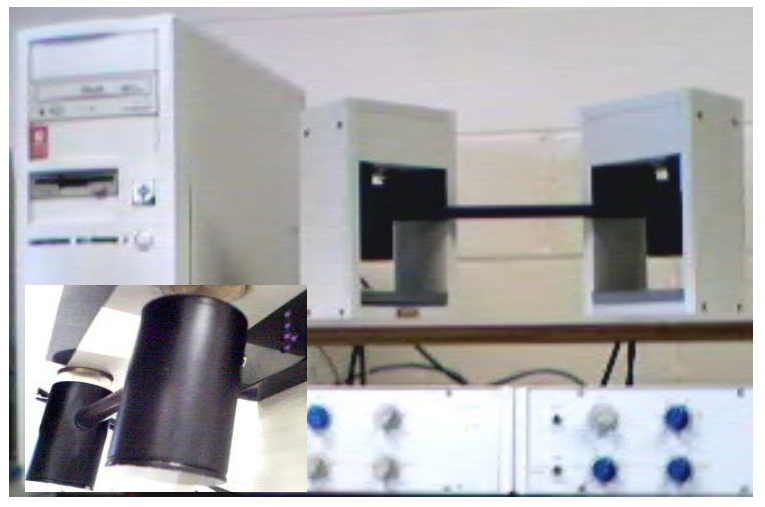

Figure 1. Overall view of the MAGLEV2 device 


\section{Linear MAGLEV Model}

The MAGLEV2 device is open-loop unstable, which limits maintaining initial stability of the suspended object while the holographic neural network is trained. This problem is handled by using a nominal linear model from the nonlinear equations of motion. The manner in which the linear equations are embedded into a neural network will be described subsequently. The MAGLEV2 is treated as 2 single degrees of freedom as shown in Figure 2. Bellow one axis is considered in details.

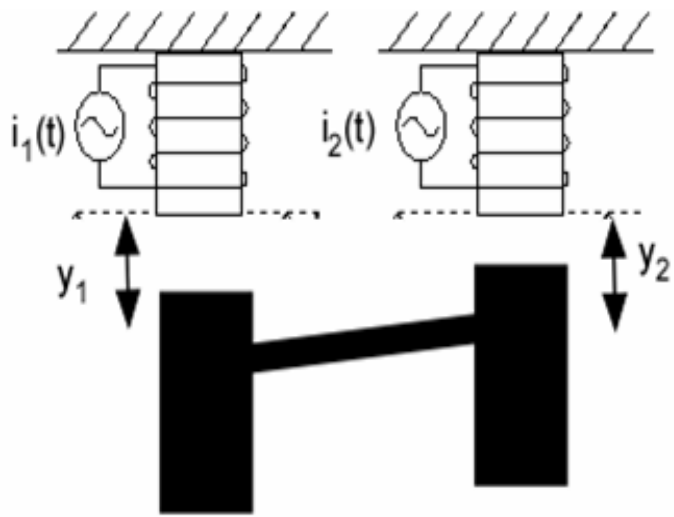

Figure 2: Coil and two cylinders connected by a rod

A metal cylinder of mass, $m$, is placed underneath the electromagnet at a distance, $y$. The current, $i$, flowing into the electromagnetic coil will generate electromagnetic force to attract the metal cylinder. The net force between the gravitational force and the electromagnetic force will cause the metal cylinder to move up or down. The metal cylinder is kept in a dynamic balance around the operating point, $16 \mathrm{~mm}$ from the bottom of the coil. The dynamic equation is given by $[2,3]$ :

$$
\ddot{y}=g-\frac{1}{m}\left(\frac{i}{a+b y}\right)^{2}
$$

where $a$ and $b$ are experimental constants and $y$ is the vertical distance from the bottom of the coil. It is an inverse square law that reduces the electromagnetic force taken to suspend the metal cylinder further from the coil. The linear model is generated by linearizing Equation (1) about a nominal operating point, $\left(\mathrm{y}_{0}, \mathrm{i}_{0}\right)$, to obtain:

$$
\ddot{y}=k\left(\frac{i_{0} b y}{a+b y_{0}}-i\right)
$$

This continuous-time model is sampled at a rate of 1 $\mathrm{kHz}$ using a zero-order hold approximation yielding the linear difference equation:

$$
\begin{aligned}
& y(n)=b_{0} i(n)+b_{1} i(n-1)+b_{2} i(n-2)+ \\
& a_{1} y(n-1)+a_{2} y(n-2)
\end{aligned}
$$

The above model is an $\operatorname{ARMA}(3,2)$ model linear in the parameters.

\section{Holographic Neural Networks}

Artificial Neural Networks (ANN) have recently emerged as a useful tool for learning a mapping from a given set of input-output associations. The network is comprised of a number of neurons organized in successive layers, with the outputs of the neurons in each layer connected by synapses of variable weights to the inputs of neurons of the next layer. Each neuron is a non-linear input-output unit with a sigmoid transfer function which, being easily differentiable, lends itself to a gradient descent method called backpropagation. The error of the output of each layer is propagated back to the previous layer, and the weights are changed in a way to decrease that error. The major problem encountered when using ANN are the prescription of the number of neurons in each layer, the number of hidden layers and the susceptibility of the gradient descent to local minima and flat spots, resulting in considerable learning time.

The Holographic Neural Networks (HNN) introduced in Sutherland [4] present a different viewpoint than that of the classical ANN. With the HNN paradigm, the input-output associations are both learned and recalled in a non-connectionist and a non-iterative transformation. The theoretical basis behind HNN design employs the mathematics of complex integers in a manner similar to holography. HNN are described in general terms in the following section. Further details are given in $[4,5]$.

A sequence of scalar input-output data fields are transformed into a set of complex vectors oriented about the origin in the complex plane and bounded within a unit circle. Each vector operates within two degrees of freedom, that is, phase and magnitude. The information content is assigned to the component of phase orientation, whereas the associated vector magnitude indicates a confidence level in that information (phase) value. The magnitude values are typically bounded within a probabilistic range (i.e. 0.0 to 1.0). It represents the weight allocated to the phase value. For phase angles to represent scalar values that extend over unrestricted range, a scaling transformation is used. The sigmoid function provides a direct means of mapping the stimulus-response data fields into a closed circular range $[0,2 \pi]$ extending 
about the complex plane. The symmetrical form of the sigmoid transformation achieves a uniform probabilistic distribution of vectors oriented about the origin in the complex plane. Detailed analysis of holographic models is given in Sutherland [\#\#\#]. Consider a set $\mathrm{S}$ of scalar values defining an input (stimulus) field. A sigmoidal preprocessing consists of the following transformation:

$$
\begin{aligned}
& s_{k} \longrightarrow \lambda_{k} e^{j \theta_{k}} \\
& \theta_{k} \longrightarrow 2 \pi\left(1+e^{\left(\mu-s_{k}\right) / \sigma}\right)^{-1}
\end{aligned}
$$

Where $\mu$ is the mean of distribution over $\mathrm{S} ; \mathrm{k}=1$ to $\mathrm{N}$, $\sigma$ is variance of distribution, and $\lambda_{\mathrm{k}}$ is the assigned confidence level. One may construct sets of stimulus and response fields extending over a period of time frame where the data element index is along the horizontal and time incremented along the vertical:

$$
[S]=\left[\begin{array}{l}
\left(\lambda_{1} e^{j \theta_{1}}, \lambda_{2} e^{j \theta_{2}}, \lambda_{3} e^{j \theta_{3}} \ldots\right)_{t 1} \\
\left(\lambda_{1} e^{j \theta_{1}}, \lambda_{2} e^{j \theta_{2}}, \lambda_{3} e^{j \theta_{3}} \ldots\right)_{t 2} \\
\cdots \\
\cdot \\
\end{array}\right]
$$

Similarly the output (response) field can be written as:

$$
[R]=\left[\begin{array}{l}
\left(\gamma_{1} e^{j \phi_{1}}, \gamma_{2} e^{j \phi_{2}}, \gamma_{3} e^{j \phi_{3}} \cdots\right)_{t 1} \\
\left(\gamma_{1} e^{j \phi_{1}}, \gamma_{2} e^{j \phi_{2}}, \gamma_{3} e^{j \phi_{3}} \cdots\right)_{t 2} \\
\cdots \\
\cdot \\
\cdot
\end{array}\right]
$$

The encoding (learning) operation involves an association between the complex representation of the stimulus and response fields and is captured within a correlation matrix. This matrix is the result of the inner product of the complex conjugate of the stimulus field and the complex response field. Each of the complex elements of the correlation matrix has a magnitude and a phase orientation. The magnitude represents a weighting of the learned input-output association extending over a unit circle. The phase is the difference between the response phase and the input phase. It defines an ability to express a component of analog response when presented with prior analog stimuli. This encoding is represented by:

$$
[X]+=[\bar{S}]^{T} \cdot[R]=\left[\begin{array}{l}
\sum_{t}^{P} \lambda_{1} \gamma_{1} e^{j(\phi 1-\theta 1)} \\
\sum_{t}^{P} \lambda_{2} \gamma_{2} e^{j(\phi 2-\theta 2)} \\
\cdots \\
\cdot
\end{array}\right]
$$

Complex multiply an operation being commutative, any phase rotational operation (complex multiplication) performed on a complex vector induces an equivalent rotation on the set of complex vectors from which it is comprised. Thus, HNN preserve information within the aggregate complex scalars superposed or enfolded with a complex summation. The objective is the recall of input-output associations stored within the complex valued correlation matrix cells.

Decoding or response recall is achieved by the complex inner product of the new input field and the correlation matrix. The prior encoded stimulus patterns displaying the greatest similarity to the new input pattern produce a predominant recognition response (in phase and magnitude). The remaining terms manifest a smaller dominance or magnitude and follow a path characteristic of random walks. The error component depends not upon any individual association but upon the relationship between the new input and the set of disassociated mapping in the correlation set. Here the response recall is represented by an iterative matrix formulation as follows:

$[R]^{1}=\frac{1}{c}[S]^{0} \cdot[X]^{0},[X]^{1}=[\bar{S}]^{0} \cdot[R]^{1},[R]^{2}=\frac{1}{c}[S]^{0} \cdot[X]^{1},(8)$ where $\mathrm{c}$ is a scaling factor. Only two or three iterations are required for convergence within a MSE of $10^{-5}$. Simple complex matrices operations are fully supported in Simulink.

\section{Holographic Neural Predictive Control}

A complete derivation of the Neural Generalized Predictive Control algorithm for a general SISO system is developed in [6]. In this application, a HNN is used instead of a classical ANN. The block diagram of the Holographic Neural Predictive Control system (HNPC) is shown in Figure 3. It consists of the MAGLEV2 device, a holographic neural network model for prediction, a trajectory of the desired 
position of the suspended cylinder, and the Cost Function Minimization (CFM) algorithm that calculates the current command needed for the magnetic coil to produce the desired position.

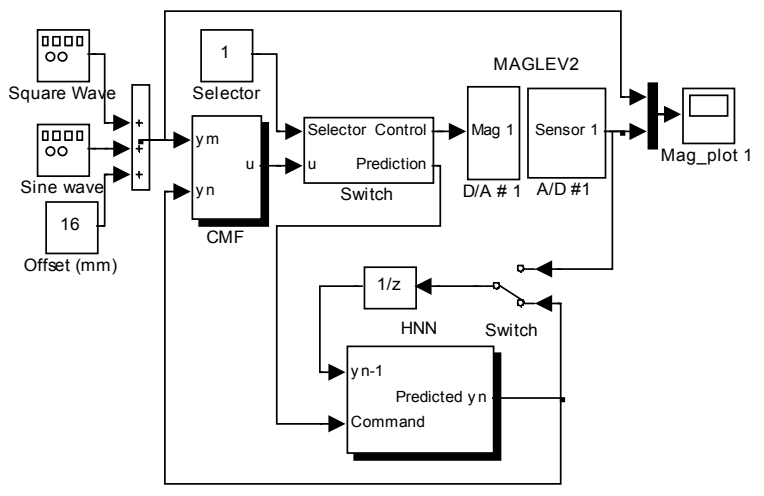

Figure 3. Simulink Model of the HNPC system.

The HNPC algorithm operates in two modes, prediction and control. Prediction occurs between samples by setting a double switch, to the HNN model to predict over some finite horizon, the response of the plant to the inputs calculated by the CFM algorithm. The CFM algorithm minimizes a user specified cost function to calculate the next control input. The system is set back to a mode of control before the next sample time when the switch is set back to the MAGLEV2 device. At this time the control input that minimizes the cost function over the entire horizon is passed to the MAGLEV as the current command, $u(n)$. The main steps are $[8,9,10]$ :

i) Starting with the previously calculated control input, $u(n)$,predict the performance of the plant for the specified horizon using the model. The value of the horizon is determined through a priori tuning.

ii) Calculate a new control input that minimizes the cost function,

iii) Repeat steps 1 and 2 until desired minimization is achieved,

iv) Send the "best" control input to the maglev as the new $u(n)$,

v) Repeat for each time step.

The cost function used for position control of the

System has three terms. The first term represents the sum of the mean square error between the desired output and the output of the neural network model. The HNN model serves to predict the plant outputs from N1 to N2 future time steps. The second term is the weighted square of the control increments. The calculated inputs for the plant predictions form the control increments. They are calculated for $\mathrm{N}_{\mathrm{u}}$ future time steps. The only constraint on the values of the horizons is that $\mathrm{Nu}$ and $\mathrm{N} 1$ be less than or equal to $\mathrm{N} 2$. $J=\sum_{j=N_{1}}^{N_{2}}[y m(n+j)-y n(n+j)]^{2}+\sum_{j=1}^{N_{u}} \lambda_{u}[\Delta u(n+j)]^{2}+g(u)(9)$ where

$\mathrm{N}_{1}$ is the minimum-costing horizon,

$\mathrm{N}_{2}$ is the maximum-costing horizon,

$\mathrm{N}_{\mathrm{u}}$ is the control horizon,

$y_{m}$ is the desired tracking trajectory,

$y_{n}$ is the predicted output of the model,

$\lambda_{\mathrm{u}}$ is the control input weighting factor,

$\Delta u(n+j)=\mathrm{u}(\mathrm{n}+\mathrm{j})-\mathrm{u}(\mathrm{n}+\mathrm{j}-1)$

$\mathrm{g}(\mathrm{u})$ defines constraints placed on the control input over a horizon of $\mathrm{N}_{\mathrm{u}} \cdot[6,7]$.

The algorithm used to minimize the cost function is the quadratic Newton-Raphson iterative algorithm. Less than five iterations were needed for convergence within an average error of $10^{-6}$. The network shown in Figure 4 depicts the structure with the linear model embedded during the training of the HNN model. The current, $i$, is the input to the MAGLEV2 system and vertical position, $y$, is the output. The inputs to the network are current, past values of the current, and the past position measurements of the MAGLEV2.

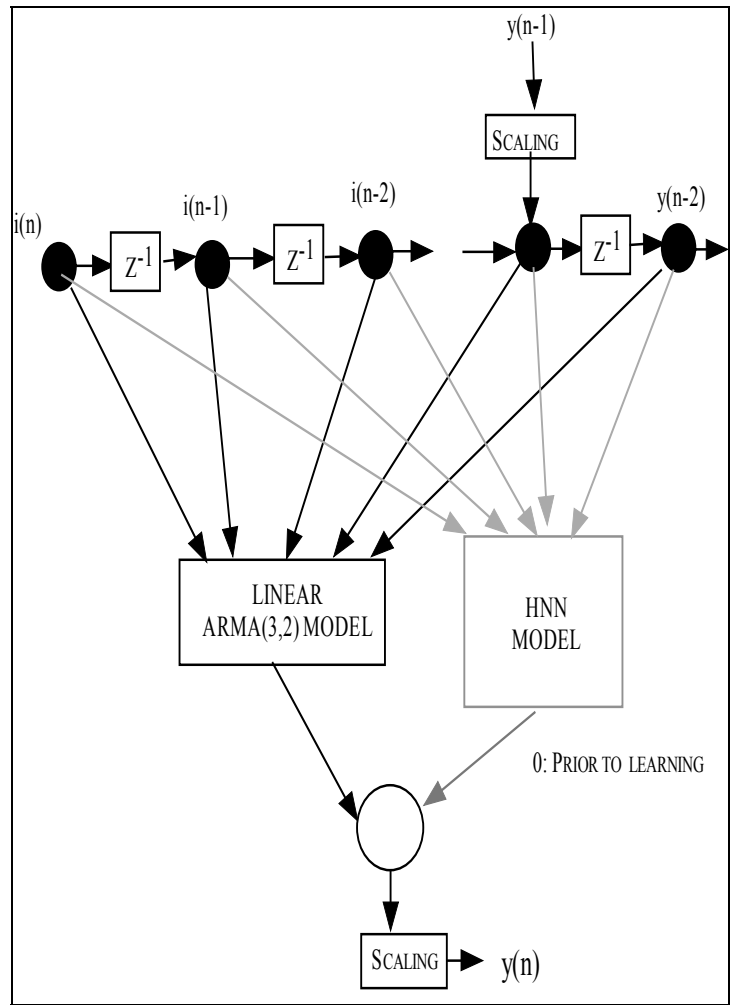

Figure 4. Network structure during training. 


\section{Results and conclusions}

The position of the cylinder is set to track a $2 \mathrm{~Hz}$ sine wave about the linearised operating point of $16 \mathrm{~mm}$. The maximum distance commanded is $\pm 5 \mathrm{~mm}$. Although, there is no systematic method to determine the values for the tuning parameters $\mathrm{N}_{1}, \mathrm{~N}_{2}, \mathrm{~N}_{\mathrm{u}}$, and $\lambda_{\mathrm{u}}$ is for a nonlinear system, their initial values were set using the general guidelines outlined in $[10,11]$. In simulation mode when the linear model is used, the parameters were: $\mathrm{N}_{1}=1$, $\mathrm{N}_{2}=6, \mathrm{~N}_{\mathrm{u}}=2$, and $\lambda=10^{-3}$. In MAGLEV2 control mode, good results were obtained when the parameters were tuned to $\mathrm{N}_{1}=1, \mathrm{~N}_{2}=14, \mathrm{~N}_{\mathrm{u}}=2$, and $\lambda_{\mathrm{u}}=0.55$. The tracking performance of one metal cylinder under a sine input is shown in Figure 5. A major reduction in the error between the HNPC and the MAGLEV2 can be seen after 35 seconds of training. The sampling time is set to $0.001 \mathrm{~s}$.

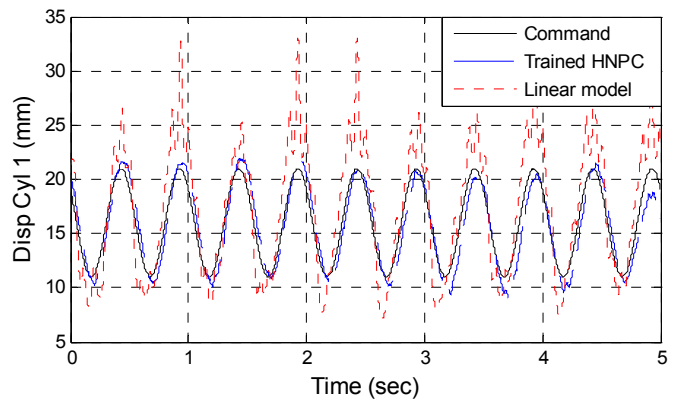

Figure 5. Trajectory tracking during HNPC training.

Further tests were conducted on the two degrees of freedom device. Figure 6a shows initial performance of the HNPC after 15 seconds training (6a), while the bottom plots (6b) shows good tracking after 50 seconds of learning. The input trajectories were a square waves with a frequency of $0.5 \mathrm{~Hz}$ and amplitude of $4 \mathrm{~mm}$ with opposite phase.

In this new set up, the use and development of an interactive real-time digital control system, using standard off-the-shelf components, the familiar software package MATLAB/SIMULINK and some of its toolboxes, a $\mathrm{C}++$ compiler, a Pentium IV $3.8 \mathrm{GHz}$ and a nonlinear maglev system as the HIL device suitable for engineering students in area of Mechatronics and Control. This PC based environment provides for the implementation of novel control algorithms using Simulink blocks and allows for interactive real-time system tuning. All system parameters, such as controller horizons, and reference inputs, can be viewed and modified dynamically; their effects can be observed on the HIL device without having to rebuild and download the new model to the target DAC. The data for any signal available in the Simulink diagram or interfaced on the DAQ card can be observed and saved in MATLAB format for analysis.
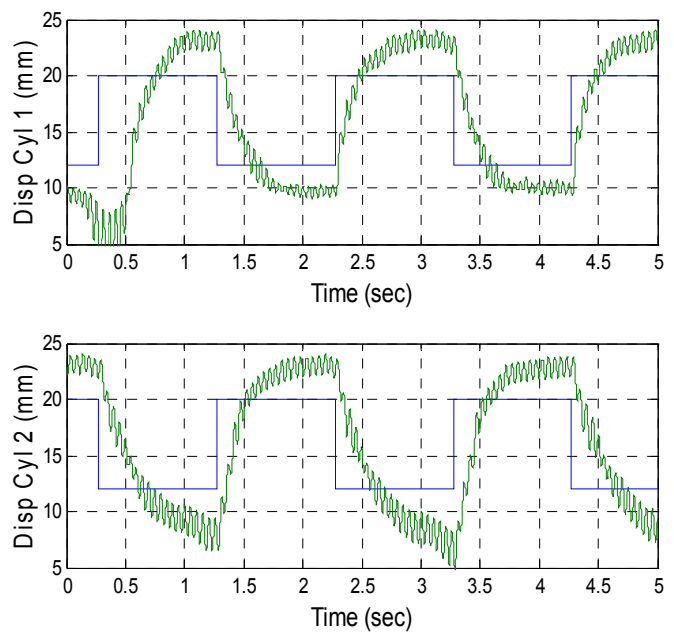

(a)
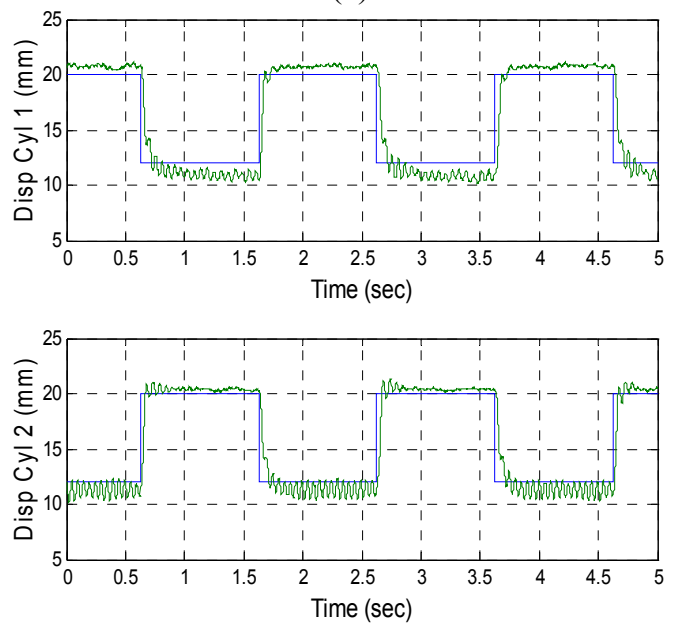

(b)

Figure 6. Tracking performance of the HNPC

\section{References}

[1] Real Time Workshop User's Guide, MathWorks, Inc., Natick, MA, 1999.

[2] P. Haley, D. Soloway, and B. Gold, "Real-time adaptive control using neural generalized predictive control," in Proc. Amer. Control Conf., San Diego, CA, June 1999, pp. 4278-4282.

[3] P. S. Shiakolas and D. Piyabongkarn, "Development of a real-time digital control system with a hardware-in-the loop magnetic levitation device 
for reinforcement of controls education," IEEE Trans. Educ., vol. 46, pp. 79-87, Feb. 2003.

[4] J.G. Sutherland, The holographic neural method. In: Soucek, B. (Ed.), Fuzzy, Holographic and Parallel Intelligence. Wiley, New York, pp. 7-92. 1992.

[5] R. Boudreau., Levesque G., et Darenfed S. "Parallel manipulator kinematics learning using holographic learning models." Robotics and Computer-Integrated Manufacturing, 14:37-44, 1998.

[6] D. Soloway and P. Haley, "Neural Generalized Predictive Control: A Newton-Raphson Implementation," Proceedings of the IEEE $C C A / I S I C / C A C S D$, IEEE Paper No. ISIAC-TA5.2, Sept. 15-18, 1996.

[7] Clarke, D.W., C. Mohtadi, and P.S. Tuffs "Generalized Predictive Control, Part I "The Basic Algorithm", Part II "Extensions and Interpretation".” Automatica, vol. 23-2, pp. 137-160, Mars. 1987a

[8] Clarke, D.W., and R. Scatollini. "Constrained Receding Horizon Predictive Control." In: Proceedings IEE-D, Vol. 138, pp. 347-354. 1991

[9] Mingsian R. Bai et Kwuen-Yieng Ou. Experimental evaluation of adaptive predictive control for rotor vibration suppression. IEEE Transactions on control systems technology, 10(6) :895-901, November 2002.

[10] Maaziz, M.K., (2000). Commande prédictive de systèmes non linéaires: Application à la commande de machines asynchrones. Thèse de Doctorat, Université Paris Sud Orsay, École Supérieure d'Électricité, Septembre.

[11] S. A. Green and K. C. Craig, "Robust, digital, nonlinear control of magnetic-levitation system," Trans. ASME, vol. 120, no. 4, pp.488-495,Dec. 1998.

\section{Appendix}

Specifications of the MAGLEV2:

Type of Levitation: Active Electromagnetic with field augmentation in both stator and levitated member.

Number of controlled Axes:

2

Maximum Operating Current: $\quad 1.5$ Amp per axis

Maximum Operating power : $\quad 35 \mathrm{~W}$ per axis
Sensor type: $\quad$ Electro-optical (Through-beam infrared)

Set-point active range: $\quad 12 \mathrm{~mm}$ Input/output port:

With External Inerface: $\begin{array}{ll}\text { Input (command) signal Range: } & 0 \text { to }+5 \mathrm{~V} \\ \text { Output (position) signal: } & 0 \text { to }+5 \mathrm{~V}\end{array}$ Dimensions:

Stator: $128 \mathrm{~mm}(\mathrm{~W}), 90 \mathrm{~mm}(\mathrm{D}), 210 \mathrm{~mm}(\mathrm{H})$

Levitated Body: two Cylindrical bodies having embedded permanent magnets.

Overall dimensions of each hollow cylinder: $40 \mathrm{~mm}$ (OD), $70 \mathrm{~mm}(\mathrm{~L})$

Weight:

Stator: $2 \mathrm{~kg}$ per axis

Levitated body: 315 gm 